\title{
Prediction of a High Temperature Bonding Condition at the Interface for the Hot-Rolled Stainless Steel Clad Plate on Rolling
}

\author{
B. Guan, ${ }^{1}$ B. Y. Chen, Y. Zang, and Q. Qin \\ School of Mechanical Engineering, University of Science and Technology, Beijing, China \\ 1niuben57@163.com
}

The stainless steel-carbon steel clad plate was investigated using the theoretical analysis of various factors influencing the high-temperature interfacial bonding during its rolling. Phenomenological prediction analysis model of interfacial bonding strength at high temperature which considers the vacuum depth, rolling temperature, and rolling reduction, was established. The specific thermal simulation experiment was designed, the bonding strength of carbon steel and stainless steel at $1000 \sim 1200^{\circ} \mathrm{C}$ and compression degree of 10 30\% was measured by a Gleeble 3500 thermal simulator, as a result, the interfacial bonding ratio was obtained. The results show that the bonding ratio is 0.5-0.65 at the experimental temperature and compression degree. The numerical simulation method was used to analyze the influence of the compression degree of the first pass for a $2000 \times 1500 \times 100 \mathrm{~mm}$ stainless steel clad plate under the interfacial bonding condition. The simulation results show that the optimum compression degree of the first pass is $15-20 \%$ at the rolling temperature of $1200^{\circ} \mathrm{C}$.

Keywords: hot-rolling, stainless steel clad plate, bonding interface, bond strength, prediction model.

Introduction. Hot-rolled stainless steel clad plate refers to a kind of laminated composite material formed by compositing the thin stainless steel clad plate onto the base plate of thick low-carbon or low-alloy steel via the hot-rolling technology. Due to good physical and chemical properties of stainless steel and excellent mechanical properties of base plate, it is widely used in various industries, including nuclear power, shipbuilding, oil pipeline construction, and so on $[1,2]$.

Forming a solid metallurgical bonding at the bimetallic interface is the key to achieve the metal rolling composite. A large number of scholars started to explore and study the influence of factors such as bimetallic interface element diffusion and production process parameters on the interfacial bonding strength from the rolling and heat treatment process [3-8]. The main research idea was to change the rolling and heat treatment process parameters based on orthogonal test to form a laminated plate sample, and then obtain the quantitative relationship between the process parameters and the final bonding strength of laminated plate sample through mechanical properties test at room temperature, and then interfacial bonding properties are explained qualitatively based on the materials science through microstructure observation or element diffusion analysis [9-14]. For example, Meng et al. [15] studied the effect of rolling and annealing temperature on the bonding properties of $\mathrm{Ag} / \mathrm{Cu}$ laminated composites. It was found that as the compression degree increased, the interfacial bonding strength increased. However, as the temperature increased, the bonding strength increased first and then decreased. Lu et al. [16] determined the optimal deformation of $\mathrm{Ag} / \mathrm{Cu}$ bonding interface through composite interface stripping force, interface morphology and composition analysis.

It can be found from the analysis of actual production process of stainless steel clad plate that first of all the large compression degree of rolling process of the first pass makes the dissimilar metal bonding interface form a reliable physical contact through a wide range of plastic deformation, as well as a certain high temperature bonding strength under the action of rolling pressure; it then gradually forms a specified shape and size in the 
subsequent rolling pass and further optimizes the structure property of the plate, and finally achieves solid metallurgical combination through the interfacial elements diffusion and re-crystallization behavior during cooling process of the plate and forms the final bonding strength. It can be seen that the effective combination of high temperature conditions during the interface hot-rolling process is the first key step to ensure the physical integrity of the interface and achieve the ultimate effective combination. However, the following often occurs in the actual production: the dissimilar metal bonding interface fails to form a good bonding state during the hot-rolling process of the first pass, resulting in the damage to the boding interface of the plate during the subsequent rolling process or loss of physical contact state, so that the plate ultimately produces a whole or partial interface delamination or non-solidified combination. Therefore, it is very important to accurately predict the high temperature bonding state of the interface during the rolling process and develop the rolling process of the metal clad plate.

As mentioned above, limited by the unclear theoretical description of the bonding mechanism of the interface and difficult in-situ observation experiments of high temperature interfacial bonding behavior, scholars mainly qualitatively speculate the high temperature bonding process of the interface during the rolling process by testing the ultimate bonding strength, binding rate, morphology and element diffusion at room temperature. In order to study the effect of hot-rolling process on the interface bonding performance and make the corresponding rolling process design, it not only requires to obtain the relationship between the process parameters and the final bonding state of the interface at room temperature, but also needs to get the quantitative high temperature bonding performance of the interface during its hot-rolling process. Some scholars have used some simple determination criteria of interface bonding state during study of roll forming process of laminated plate with the numerical simulation [17-19], but often based on the threshold of setting interface "bonding." As this simple determination criterion cannot quantitatively evaluate the high temperature bonding performance of the interface due to its lack of appropriate experimental data support, it is difficult to effectively analyze the effect of its rolling process on the bonding state of the interface.

In order to accurately describe and predict the interface bonding state of the stainless steel clad plate during the rolling process, the phenomenological predictive analysis model of interface high temperature bonding performance was established in this paper based on the theoretical analysis of various factors influencing the high temperature bonding performance of the interface during the rolling process. And the specific thermal simulation experiment was designed. The high temperature bonding performance of the stainless steel-carbon steel clad plate during the rolling process was determined experimentally. Finally, based on the experimental data, the numerical simulation method was used to study the effect of different rolling processes of the stainless steel clad plate on the interface bonding state.

1. Analysis of Influencing Factors and Establishment of Model. During the hot-rolling process of the stainless steel clad plate, the dissimilar metals are stacked in the order of $A-B-B-A$ (as shown in Fig. 1, $A$ is the base layer, $B$ is the cladding layer, and the $B-B$ layer metals are separated with the glass powder). Around the slab is welded with edge strip and left with pores, and the air inside the assemble is extracted through the pores to form a certain degree of vacuum in the assemble. Using this method can make the metal of the bonding interface during the high temperature hot-rolling process does not oxidize, thereby improving the interface bonding performance. According to the theory of metal solid state connection, the main factors influencing the interfacial bonding strength during rolling are vacuum depth, temperature and compression degree.

1.1. Effect of vacuum depth on Interfacial Bonding Strength. In the process of rolling and pre-heating, the metal atoms of bonding interface will combine the oxygen atoms in the air to form a brittle oxide, which will weaken the interfacial bonding strength 


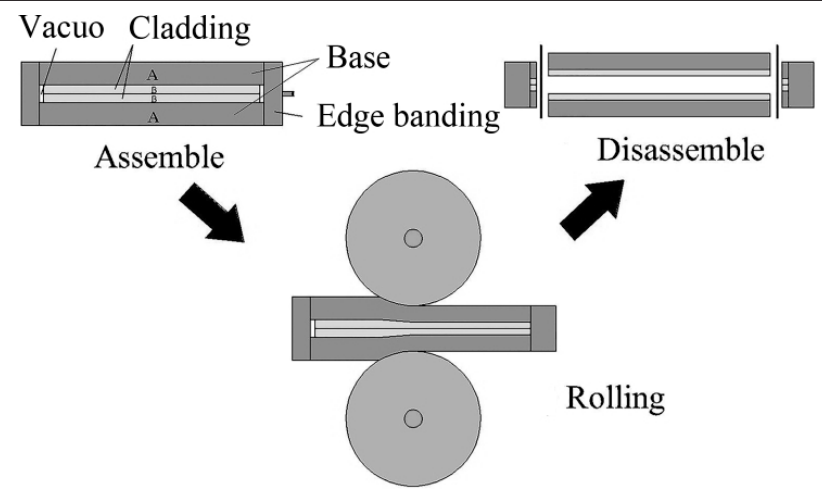

Fig. 1. Schematic diagram for assemble structure and clad plate production.

at both room temperature and high temperature, and the size of the vacuum depth directly determines the concentration of oxygen atoms at the bonding interface, therefore, the vacuum depth directly affects the bonding strength of the interface.

$\mathrm{Li}$ et al. [20] studied the effect of interfacial vacuum depth on the interfacial bonding strength of stainless steel-carbon steel by experiments. The experiments were carried out using the vacuum degrees of $0.1,1,20,2500,25,000$, and $100,000 \mathrm{~Pa}$, respectively. When the slab was heated to $1200^{\circ} \mathrm{C}$, the interfacial bonding strength at room temperature measured after 7 rolling passes (20\% of the reduction for each pass) and air cooling was as shown in Table 1. It can be known from the gas molecular dynamics that the interface metal oxide has a Boltzmann function in relation to the oxygen concentration. Therefore, the Boltzmann function is used to fit the relationship between the interfacial bonding strength and the vacuum depth. In order to allow that the function relationship can be used under any temperature condition, the bonding strength when the vacuum depth is $0.1 \mathrm{~Pa}$ is treated as the maximum value of the bonding strength, and the ratio $K_{p}$ of the interfacial bonding strength to the maximum value under each vacuum condition is obtained in the fitting process. $K_{p}$ is the influence coefficient of the interfacial vacuum depth on the interfacial bonding strength, which is taken as the criterion of the influence of different vacuum degrees on the interfacial bonding. The relationship between $K_{p}$ and pressure $P$ is shown in Fig. 2, and the function relationship obtained by fitting is given by the following equation:

$$
K_{P}=0.12+1.08\left[1+\exp \left(\frac{P-5400}{3600}\right)\right] .
$$

It can be seen that at a vacuum depth of less than $100 \mathrm{~Pa}$, the value of $K_{p}$ is close to 1 , the bonding strength of the interface is not substantially affected by the vacuum depth. The bonding strength of the interface between 100 and $10,000 \mathrm{~Pa}$ is drastically reduced to near zero. Since the vacuum depth of the assemble is often controlled at about $1 \mathrm{~Pa}$ in the actual production process of the clad plate, the value of $K_{p}$ can be regarded as 1 in the actual production condition of the stainless steel clad plate.

1.2. Effect of Rolling Temperature on Interfacial Bonding Strength. There are two main aspects of the influence of temperature on the interfacial bonding strength. Firstly, it changes the diffusion rate of the elements between the bonding interfaces [21]. Secondly, it changes the flow stress of the base and the cladding metal. The influence of the first aspect is the direct effect of the temperature on the interfacial bonding state, and the second aspect is to influence the interface bonding state by changing the mechanical properties of metal, which is the indirect influence of temperature on the interfacial bonding state. They will be discussed in Subsection 1.4. 
B. Guan, B. Y. Chen, Y. Zang, and Q. Qin

$\mathrm{T}$ a b 1 e 1

Effect of Vacuum Depth on Interfacial Strength

\begin{tabular}{|c|c|c|}
\hline Vacuum depth/Pressure, Pa & Interfacial strength, MPa & $K_{p}$ \\
\hline 0.1 & 440 & 1 \\
\hline 1 & 435 & 0.9886 \\
\hline 20 & 435 & 0.9886 \\
\hline 2500 & 280 & 0.6364 \\
\hline 25,000 & 50 & 0.1136 \\
\hline 100,000 & 50 & 0.1136 \\
\hline
\end{tabular}

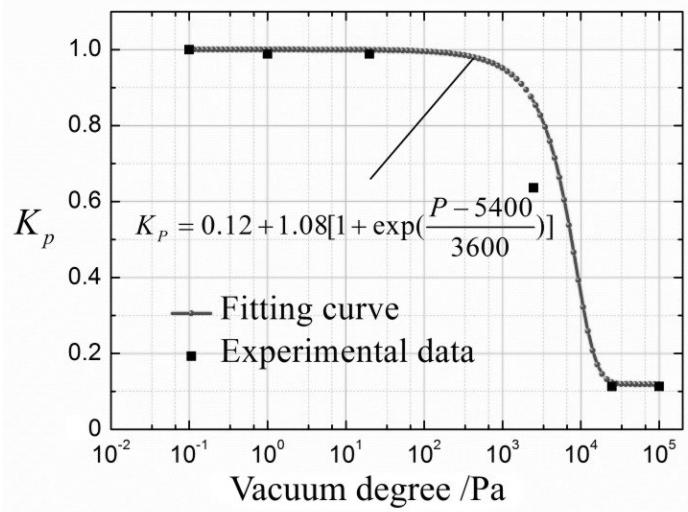

Fig. 2. Effect of vacuum depth on interfacial strength.

In terms of the effect of rolling temperature on the diffusion rate of elements, it is different from the diffusion connecting process of the general solid metal. Since the duration of the high temperature rolling process is very short, the element diffusion rate between the interfaces is not sufficient, and the diffusion region is formed only in the interface minimum range. Therefore, the influence of the diffusion of interface elements on the concentration of metal elements on both sides can be neglected in the analysis of the interfacial bonding strength at high temperature during the rolling process. It can be considered that the concentration of the elements on both sides of the interface is similar to that of the matrix material, and the mechanical properties of the metal on both sides of the interface will not change greatly due to the diffusion of elements.

1.3. Effect of Compression Degree on Interfacial Bonding Strength. According to the pressure welding theory [22-24], during the high temperature rolling process, the metal slab squeezes the bonding interface under the action of two rollers to produce a wide range of plastic deformation at the bonding interface. In this state, the mesoscopic surface morphology of the bonding interface changes and the original bimetallic interface forms an effective physical contact, and effective physical contact area of the original interface increases as the increases of plastic deformation (as shown in Fig. 3 below). According to the joint re-crystallization theory $[25,26]$, since the two metals are above the re-crystallization temperature, the area where the bimetal forms an effective physical contact on the bonding interface will undergo a joint re-crystallization during the rolling process to form a bonding interface of metallurgical bonding. This mechanism is the most important way for the bimetallic interface to form a certain bonding strength during the high temperature rolling process, where the interfacial elements are not diffused sufficiently and the static re-crystallization time is very short [27]. 


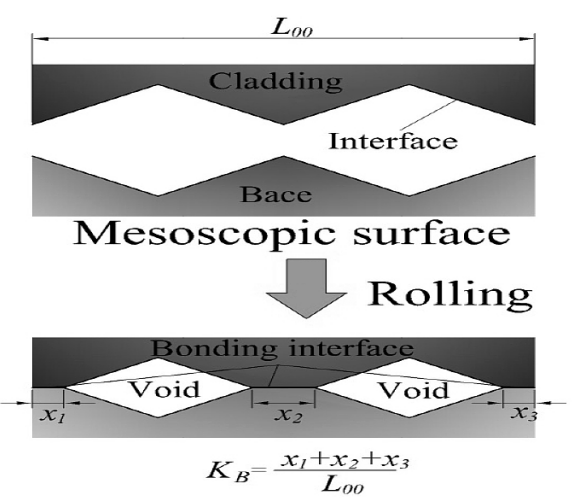

Fig. 3. Schematic diagram for interfacial bonding process at mesoscale.

The above analysis shows that the compression degree mainly affects the actual physical contact area between the two sides of the metal by changing the mesoscopic surface morphology of the bonding interface. It can be described using the interface binding rate $K_{B}$. The physical meaning of $K_{B}$ is shown in Fig. 3, which refers to the ratio of the metallurgical bonding area of the bonding interface to the geometric area of the bonding interface.

1.4. Establishment of Prediction Model for Interfacial Bonding Strength. According to the analysis made in Subsections 1.1-1.3, it can be seen that the interfacial bonding strength $\sigma_{b}$ is determined by the vacuum depth $P$, the temperature $T$, and the compression degree $\varepsilon$. From the analysis of Subsection 1.1, it can be seen that the influence coefficient $K_{p}$ of vacuum depth will affect the bonding strength of the whole interface. Therefore, the relationship between the three influencing factors and the $\sigma_{b}$ is

$$
\sigma_{b}=f(P, T, \varepsilon)=f(P) f(T, \varepsilon)=K_{p} f(T, \varepsilon) .
$$

The area where the actual physical contact is formed between the interfaces has a solid metallurgical bonding due to the joint recrystallization, and its strength property is between the two metals, which is stronger than the weaker one. So, if the bonding interface is destroyed by external forces, the delamination damage of interface will appear on the side of metal with the weaker flow stress (as shown in Fig. 4). Therefore, as for the area where the actual physical contact is formed between the interfaces, its bonding strength is determined by the flow stress $\sigma_{\text {flow }}$ of the metal with the weaker flow stress under this condition $[28,29]$. But, according to Subsection 1.3, not all the interface forms actual physical contact. The proportion of actual physical contact area to the total interface area is $K_{B}$, which is related to compression degree $\varepsilon$. So, $\sigma_{b}=K_{B} \sigma_{\text {flow }}$, if the effect of vacuum depth is neglected.

By substituting the relationship among $\sigma_{\text {flow }}, K_{B}$, and $\sigma_{b}$ into Eq. (2), namely:

$$
\sigma_{b}=K_{p} K_{B} \sigma_{\text {flow }}
$$

where $K_{p}$ is related to vacuum depth $P$ of the interface, $K_{B}$ is related to the compression degree $\varepsilon$, and $\sigma_{\text {flow }}$ represents the flow stress of the metal with weaker flow stress in the two metals, which is related to the rolling temperature $T$ and the strain rate $v$. We can get the following equation by expressing the above relation in Eq. (3):

$$
\sigma_{b}=K_{p}(P) K_{B}(\varepsilon) \min \left(\sigma_{B}(T, v), \sigma_{C}(T, v)\right) .
$$



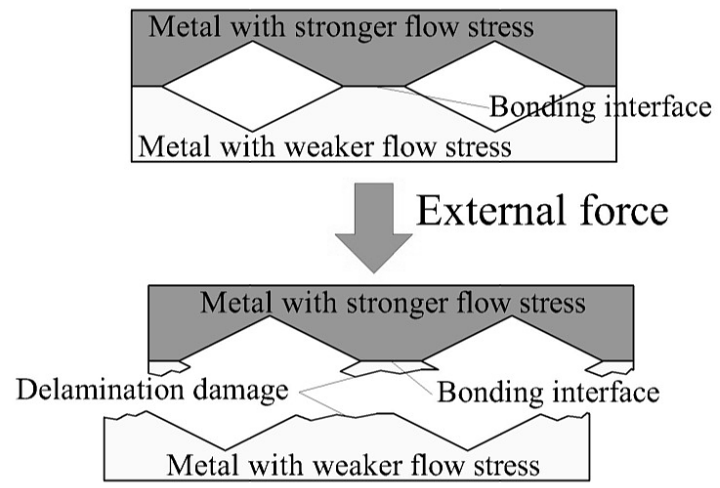

Fig. 4. Schematic diagram for interfacial damage process at mesoscale.

In Eq. (4), $\sigma_{B}$ and $\sigma_{C}$ are the flow stresses of the base metal and the cladding metal. Equation (4) is the phenomenological prediction model of the interfacial high temperature bonding strength. Since the effect of element diffusion on the interfacial bonding strength during the rolling process is neglected, it is also assumed that the bonding strength of the effective contact area of the interface is the flow stress of the metal with the weaker flow stress, so the model gets the lower limit of the interfacial bonding strength.

In order to use this model to predict the high temperature bonding strength, it not only requires to obtain the flow stresses of the base and cladding materials at different temperatures $T$ and strain rate $v$, but also needs to determine interfacial bonding ratio $K_{B}(\varepsilon)$ at the different compression degrees through specific thermal simulation experiment method.

2. Experimental Details. Based on the analysis of Subsection 1.1, $K_{p}(P)$ is close to constant 1 during the actual production process of stainless clad plate. So it only needs to design the strength test experiment of high temperature bonding interface which can determine the coefficient $K_{B}(\varepsilon)$.

2.1. Experimental Materials and Specimens. Table 2 shows the chemical compositions of Q345R carbon steel (CS) and 316L stainless steel (SS) used in this study. The specimen is obtained from plate casting billet using a wire cutting process. The cylindrical specimens with the diameter of $10 \mathrm{~mm}$ and the length of $55 \mathrm{~mm}$ are machined for bonding. The surface roughness $R a$ of specimens' end faces is approximately 1.6 3.2 $\mu \mathrm{m}$, which is close to the initial morphology state of the interface in the actual production process (Fig. 5a and b).

$\mathrm{T}$ a b 1 e 2

Element Composition of Materials

\begin{tabular}{||c|c|c|c|c|c|c||}
\hline \multirow{2}{*}{ Steel } & \multicolumn{7}{|c||}{ Element (mass fraction \%) } \\
\cline { 2 - 7 } & $\mathrm{C}$ & $\mathrm{Si}$ & $\mathrm{Mn}$ & $\mathrm{P}$ & $\mathrm{S}$ & $\mathrm{Cr}$ \\
\hline Q345R (base) & 0.17 & 0.27 & 1.40 & 0.011 & 0.007 & 0.023 \\
\hline 316L (cladding) & 0.017 & 0.42 & 1.26 & 0.031 & 0.003 & 16.030 \\
\hline & $\mathrm{Ni}$ & $\mathrm{Mo}$ & $\mathrm{Al}$ & $\mathrm{Ti}$ & \multicolumn{2}{c||}{$\mathrm{Fe}$} \\
\hline Q345R (base) & 0.02 & $<0.01$ & $<0.01$ & $<0.01$ & \multicolumn{2}{c|}{ Bal. } \\
\hline 316L (cladding) & 10.03 & 1.95 & $<0.01$ & $<0.01$ & \multicolumn{2}{c}{ Bal. } \\
\hline
\end{tabular}




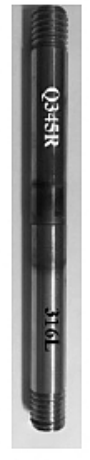

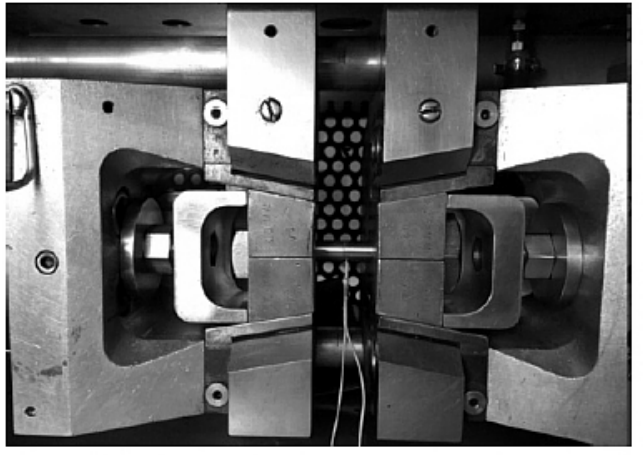

b

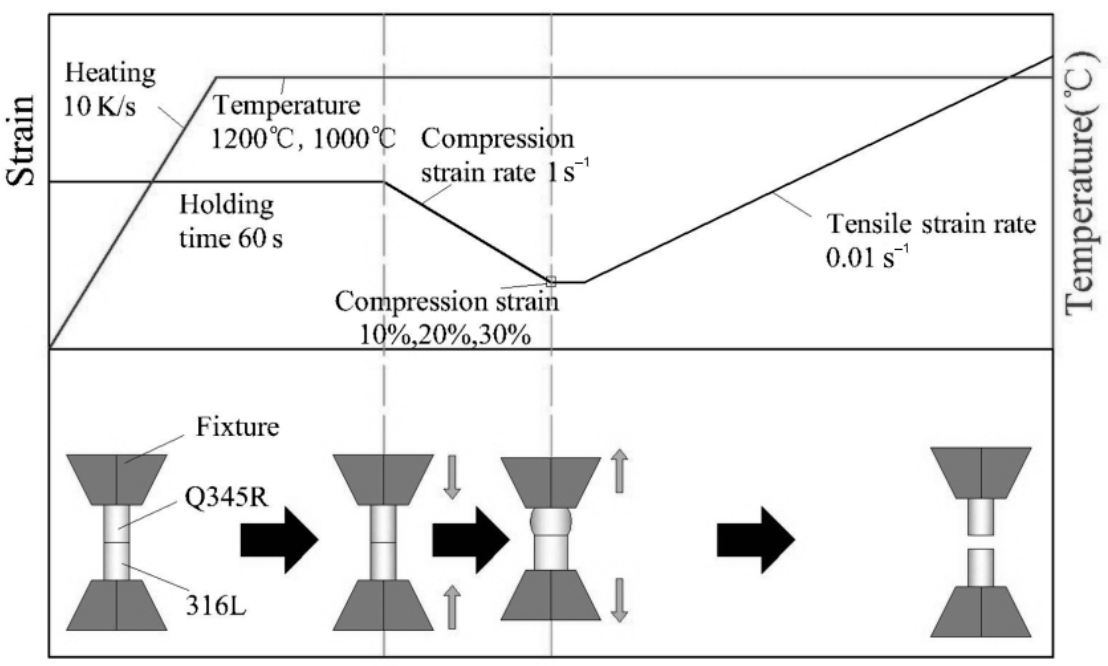

$\mathrm{c}$

Fig. 5. Experiment of bonding strength of high temperature bonding interface: (a) specimen; (b) specimen clamping; (c) schematic diagram of the experimental process.

2.2. Experimental Procedure and Parameters. The experiments were conducted using a Gleeble 3500 materials simulator. The experimental realization is shown in Fig. 5c.

During the experiment, firstly, the experimental environment is set to reach the setting vacuum depth. Then, the two specimens are brought into contact with each other and applied with a pre-stressed of about $0.1 \mathrm{MPa}$. These are used to ensure that the two specimens are in good contact during the electric heating. The two specimens are then heated to the deformation temperature and held for $60 \mathrm{~s}$ to eliminate temperature gradient in the specimens and the heating rate is $10 \mathrm{~K} / \mathrm{s}$.

Secondly, the two specimens are compressed together under the given strain rate to reach the corresponding compression degree. This part of experiment can simulate the stress and strain state that the bonding interface has experienced during the actual rolling process.

Finally, the specimen displacement remains unchanged for about $3 \mathrm{~s}$ to make the materials simulator reach a stable state. And then the two specimens are tensioned reversely under the strain rate of $0.01 \mathrm{~s}^{-1}$ until the bonding interface separates from each other. The extracted tensile stress at this point is precisely the interface bonding strength. Through this part of experiment, the interface high temperature bonding strength can be obtained. 
Based on the vacuum depth requirements in actual production process of clad plate, the vacuum depth pressure during the experimental process is set as $1 \mathrm{~Pa}$. According to the numerical simulation of the rolling process of the clad plate [18], strain rate of interface is $0.5 \sim 1.5 \mathrm{~s}^{-1}$. The average strain rate is $1 \mathrm{~s}^{-1}$. Therefore, the strain rate of the specimens during the experiment is set to $1 \mathrm{~s}^{-1}$. The hot compression process is carried out in the temperature of 1000 and $1200^{\circ} \mathrm{C}$, which is similar to the temperature of the clad plate at the rolling process of the first pass. The corresponding compression degree is 10,20 , and $30 \%$. The specific experimental parameters are shown in Table 3.

$\mathrm{T}$ a b 1 e 3

Experimental Parameters of Bonding Strength of High Temperature Bonding Interface

\begin{tabular}{|c|c|c|c|c|}
\hline $\begin{array}{c}\text { Vacuum } \\
\text { degree, } \mathrm{Pa}\end{array}$ & $\begin{array}{l}\text { Heating rate, } \\
\mathrm{K} / \mathrm{s}\end{array}$ & $\begin{array}{l}\text { Holding time, } \\
\text { s }\end{array}$ & $\begin{array}{c}\text { Compression } \\
\text { strain rate, } \mathrm{s}^{-1}\end{array}$ & $\begin{array}{c}\text { Tensile strain } \\
\text { rate, } \mathrm{s}^{-1}\end{array}$ \\
\hline 1 & 10 & 60 & 1 & 0.01 \\
\hline
\end{tabular}

2.3. Experimental Results and Discussion. Equation (3) shows that the bonding ratio $K_{B}(\varepsilon)$ of the bimetallic surface can be expressed as

$$
K_{B}=\frac{\sigma_{b}}{K_{p} \sigma_{\text {flow }}} .
$$

As $K_{p}$ is close to unity under the test conditions, Eq. (5) can be reduced to

$$
K_{B}=\frac{\sigma_{b}}{\sigma_{\text {flow }}} .
$$

The interfacial bonding strength $\sigma_{b}$ under different experimental conditions can be obtained through the above experimental process. The flow stress of Q345R carbon steel and 316L stainless steel under different conditions can also be obtained by Gleeble thermal simulation experiment [18], and then the flow stress $\sigma_{\text {flow }}$ of the metal with weaker flow stress under different experimental conditions can be obtained. Therefore, the bonding ratio $K_{B}(\varepsilon)$ of the bimetallic interface under different compression degree conditions can be calculated by Eq. (6). The effect of process parameters on $\sigma_{b}$ and $K_{B}(\varepsilon)$ are shown in Table 4 and Fig. 6.

$\mathrm{T}$ a b 1 e 4

Experimental Results of High Temperature Bonding Strength for Binding Interface

\begin{tabular}{||c|c|c|c|c||}
\hline $\begin{array}{c}\text { Temperature, } \\
{ }^{\circ} \mathrm{C}\end{array}$ & $\begin{array}{c}\text { Compression } \\
\text { degree } \varepsilon, \%\end{array}$ & $\begin{array}{c}\sigma_{\text {flow }}, \\
\mathrm{MPa}\end{array}$ & $\begin{array}{c}\sigma_{b}, \\
\mathrm{MPa}\end{array}$ & $K_{B}$ \\
\hline \multirow{3}{*}{1200} & 10 & 52.7 & 29.0 & 0.55 \\
\cline { 2 - 5 } & 20 & 63.0 & 37.8 & 0.60 \\
\cline { 2 - 5 } & 30 & 58.4 & 36.8 & 0.63 \\
\hline \multirow{2}{*}{1000} & 10 & 95.2 & 47.6 & 0.50 \\
\cline { 2 - 5 } & 20 & 114.5 & 65.3 & 0.57 \\
\cline { 2 - 5 } & 30 & 123.3 & 80.1 & 0.65 \\
\hline
\end{tabular}




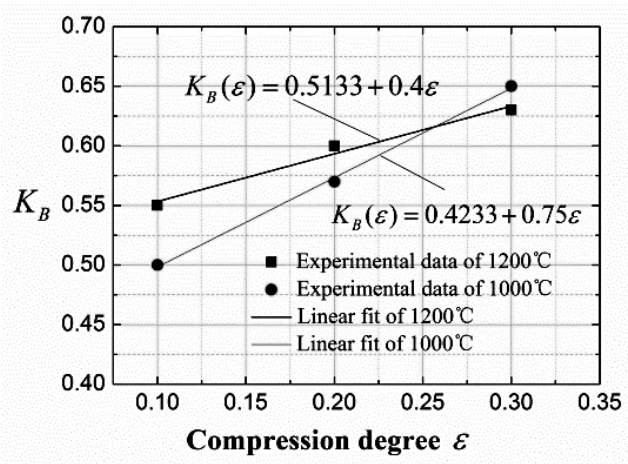

a

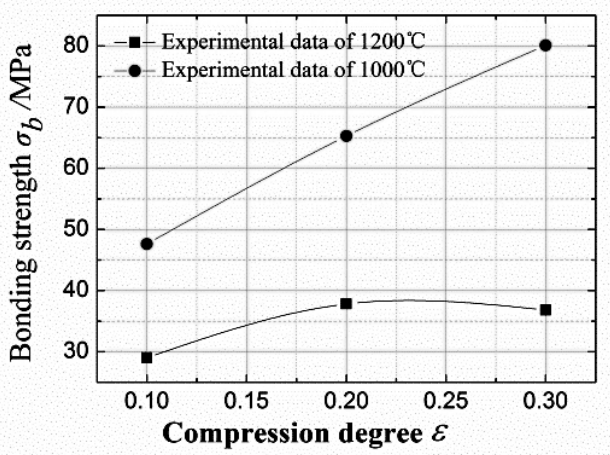

b

Fig. 6. Experimental results of high temperature bonding strength for bonding interface: (a) $K_{B}(\varepsilon)$ changes with rolling reduction; (b) $\sigma_{b}$ changes with rolling reduction $\varepsilon$.

It can be seen that with the increase of the compression degree, the bonding strength $\sigma_{b}$ of the interface does not necessarily show the monotonically increasing law affected by the change of the flow stress $\sigma_{\text {flow }}$ of the material. For example, under the temperature of $1200^{\circ} \mathrm{C}$, the bonding strength $\sigma_{b}$ under the compression degree of $20 \%$ is higher than that of $30 \%$. It can be explained as follows. According to Eq. (3), $\sigma_{b}$ is co-determined by $\sigma_{\text {flow }}$ and $K_{B}$. Though $K_{B}$ increases with the compression degree, $\sigma_{\text {flow }}$ is reduced. So $\sigma_{b}$ declines with the increase of compression degree. The interfacial bonding ratio $K_{B}(\varepsilon)$ will increase with the increase of the compression degree $\varepsilon$, and $\varepsilon$ is approximately linear with $K_{B}(\varepsilon)$. By comparing with the change law of $\sigma_{b}$ and $K_{B}(\varepsilon)$ at different temperatures, it can be found that the value of the interfacial bonding strength $\sigma_{b}$ is obviously higher at the lower temperature of $1000^{\circ} \mathrm{C}$, and the value of $K_{B}(\varepsilon)$ is basically the same. This phenomenon can be explained based on the interface contact model of Subsection 1.3. That is, at any temperature condition, as long as the compression degree is the same, the area where the interface forms the effective bonding is basically the same, so the value of $K_{B}(\varepsilon)$ is basically the same. But the strength of the material in the effective bonding area is different under different temperatures and compression degrees, and the strength of the material under the low temperature condition is higher than that of the high temperature, so the bonding strength $\sigma_{b}$ is higher under the low temperature condition.

3. Numerical Simulation. The analysis of the stress evolution process of the bonding interface during the rolling process shows that the bonding interface can form certain strength under the action of plastic deformation during the rolling process. And in the rolling process, a continuous and synergistic plastic deformation will be produced between the dissimilar metals by transferring the load through the bonding interface. However, due to the different plastic deformation characteristics of dissimilar metals and the deformation characteristics of the rolling process itself, the bonding interface will be subject to a greater shear stress during the rolling process. If this shear stress exceeds the bonding strength of the interface, the bonding state of the bonding interface will be destroyed and cause delamination damage of the metal clad plate [17]. During the actual production process, the first pass rolling is most likely to produce the damage to the interface. This is because, on the one hand, at this time the temperature of the assemble is still high, and the interface high temperature bonding strength is low; on the other hand, for the first pass, deformation rate of single pass is often larger, and the shear stress and shear strain at the bonding interface are larger. In the subsequent rolling pass, with the decrease of the assemble temperature and single pass deformation rate, the interfacial bonding strength will increase, while the shear stress and shear strain will be reduced, therefore, it is difficult to be destroyed. 
In this section, the finite element (FE) model is established to carry out the numerical simulation for the rolling process of the first pass of the stainless clad plate. Then the bonding strength prediction model established in section 1 and the experimental data obtained in Section 2 are used to predict the bonding and damage state of the high temperature interface.

3.1. Establishment of 3D FE Model. The model was established in ABAQUS explicit software by using the size parameter of stainless steel clad plate assembles in a steel plant in China. The overall size of the assemble is $2000 \times 1500 \times 200 \mathrm{~mm}$ in length, width, and height, in which the thickness of a single Q345R base slab is $90 \mathrm{~mm}$, the thickness of a single $316 \mathrm{~L}$ cladding slab is $10 \mathrm{~mm}$, and the width of the edge strip of the packaged assemble is $30 \mathrm{~mm}$. A quarter models of the plate and the roller was built because of the symmetry of the structure, as shown in Fig. 7. The material properties of the base Q345R and the cladding $316 \mathrm{~L}$ were based on the experimental data provided in [18].

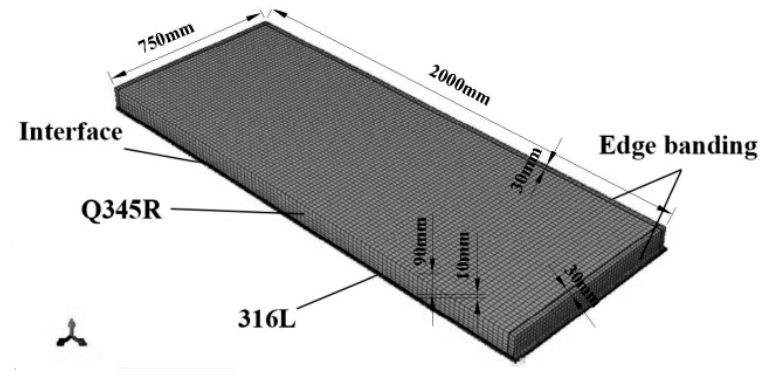

a

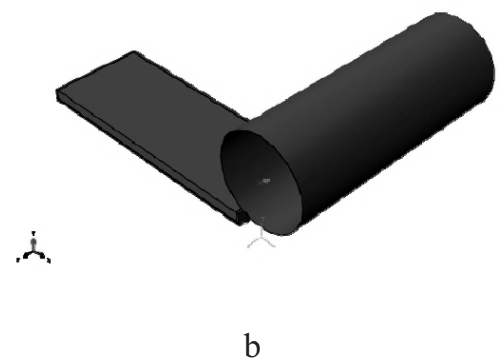

b

Fig. 7. Finite element rolling model: (a) finite element grid; (b) rolling process model.

Element type C3D8RT was chosen to mesh the plate assemble. Interface between different metals shared the same nodes. Through trial computation, the element size was set as about $15 \mathrm{~mm}$ and there were a total of 200,000 elements in this model. The roller diameter is $750 \mathrm{~mm}$. It was assumed to be analytical rigid bodies in order to reduce the solution time. In the model, according to the actual production conditions, the initial temperature of the plant assemble is set as $1200^{\circ} \mathrm{C}$ and the initial roll temperature is $300^{\circ} \mathrm{C}$. The roll speed is $0.424 \mathrm{rad} / \mathrm{s}$, that is, the linear velocity of roll rotation is about $1000 \mathrm{~mm} / \mathrm{s}$.

The symmetrical displacement constraint was applied to the symmetry area of the quarter model. The penalty friction model was applied to simulate the contact between the roller and plates assemble. The friction coefficient was defined as 0.3 . The convective heat transfer coefficient of the plate surface was set as $10 \mathrm{~W} /\left(\mathrm{m}^{2} \cdot \mathrm{K}\right)$, the rate of emission of radiation was 0.8 , and the contact heat transfer coefficient between the roller and the plates assemble was defined as $20 \mathrm{~kW} /\left(\mathrm{m}^{2} \cdot{ }^{\circ} \mathrm{C}\right)[30,31]$.

In order to improve the solution efficiency of numerical simulation, the mass scaling factor of the model is determined as 100 according to the results of the trial, then the calculation time is shortened significantly, and the rolling force fluctuation range is small. Using this model to simulate the rolling process of the first pass under 10, 15, 20, 25, and $30 \%$ total of 5 different compression degree conditions.

3.2. Shear Stress Distribution of Bonding Interface. As mentioned earlier, the main cause of interface damage during the rolling process is the delamination failure caused by shear stress. Therefore, in the study of interface bonding and damage state, the shear stress $\tau_{x y}$ of the interface parallel to the rolling direction is extracted as a main parameter to judge the damage. 
According to the analysis of Mohr circle, when the interface undergoes damage, the maximum shear stress $\tau_{b}$ that the interface withstand is 0.5 times its normal stress (i.e., bonding strength $\sigma_{b}$ ), that is

$$
\tau_{b}=0.5 \sigma_{b}
$$

So an approximate relation can be established, namely, the tangential bonding strength $\tau_{b}$ of the interface is 0.5 times the vertical bonding strength $\sigma_{b}$ of the interface. When determining the interface damage, the shear stress $\tau_{x y}$ of the bonding interface during the rolling process is extracted to compare with its tangential bonding strength $\tau_{b}$ to determine the damage of the interface.

Taking the compression degree of the first pass rolling as $20 \%$ (as an example), the distribution of the shear stress of the bonding interface during the rolling process is shown in Fig. 8. It can be seen that there are two large shear stress regions of path-1 and path-2 for the bonding interface during the rolling process, which are located at the entrance and exit sides of the roll, respectively. According to the study of reference [17], in the actual production process, the two metals at the entrance side path-1 of the roll have not yet completed the bonding, and the shear stress at the bonding interface can increase the plastic deformation of the bonding interface and promote the interface bonding ratio. And at the exit side path-2, the interface has been completed high temperature bonding, where the shear stress at this time is likely to cause bonding interface damage. Therefore, in the subsequent analysis, the path-2 region at the exit side of the roll is used as the determination area where the bonding interface is damaged.

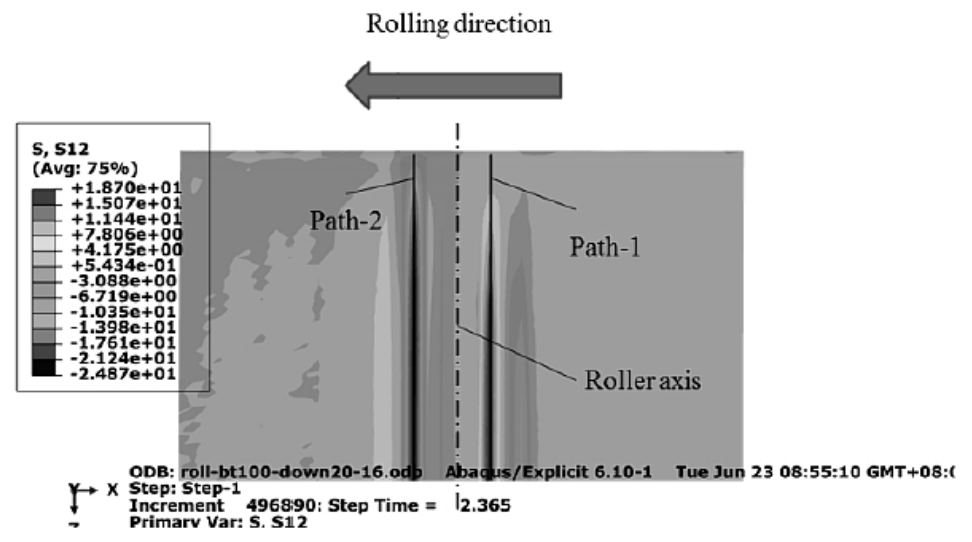

Fig. 8. Shear stress distribution of bonding interface during the rolling process.

3.3. Analysis of Bonding and Damage State of Interface. According to the fitting formula provided by Fig. $6 \mathrm{a}$, the bonding ratio $K_{B}$ of the bonding interface under each compression degree can be obtained. According to the flow stress data of the base Q345R and the cladding $316 \mathrm{~L}$ provided by the reference [18], $\tau_{b}$ can be obtained by Eqs. (3) and (7), as shown in Table 5.

Compare the shear stress at path-2 under each compression degree with, as shown in Fig. 9. During the steady rolling process, at the same position in the width direction, the stress strain state of the plate can be considered to be completely consistent along the length direction. So if the shear stress of the bonding interface at certain position in the width direction exceeds $\tau_{b}$, the total bonding interface along the length direction of the plate at the position in the width direction can all be considered to be damaged. So, by calculating the porportion of the damaged line segment in the width direction to the whole width, the proportion $K_{\text {damage }}$ of the damage area of the bonding interface to the total bonding 
$\mathrm{T}$ a b 1 e 5

Tangential Bonding Strength $\tau_{b}$ of Bonding Interface under Different Compression Degrees

\begin{tabular}{||c|c|c|c|c|c||}
\hline \hline $\begin{array}{c}\text { Temperature, } \\
{ }^{\circ} \mathrm{C}\end{array}$ & $\begin{array}{c}\text { Compression } \\
\text { degree } \varepsilon, \%\end{array}$ & $K_{B}$ & $\begin{array}{c}\sigma_{\text {flow }}, \\
\mathrm{MPa}\end{array}$ & $\begin{array}{c}\sigma_{b}, \\
\mathrm{MPa}\end{array}$ & $\begin{array}{c}\tau_{b}, \\
\mathrm{MPa}\end{array}$ \\
\hline \multirow{3}{*}{1200} & 10 & 0.55 & 52.7 & 29,0 & 14.5 \\
\cline { 2 - 6 } & 15 & 0.57 & 60.4 & 34.6 & 17.3 \\
\cline { 2 - 6 } & 20 & 0.60 & 63.0 & 37.8 & 18.9 \\
\cline { 2 - 6 } & 25 & 0.61 & 62.0 & 38.0 & 19.0 \\
\cline { 2 - 6 } & 30 & 0.63 & 58.4 & 36.8 & 18.4 \\
\hline
\end{tabular}

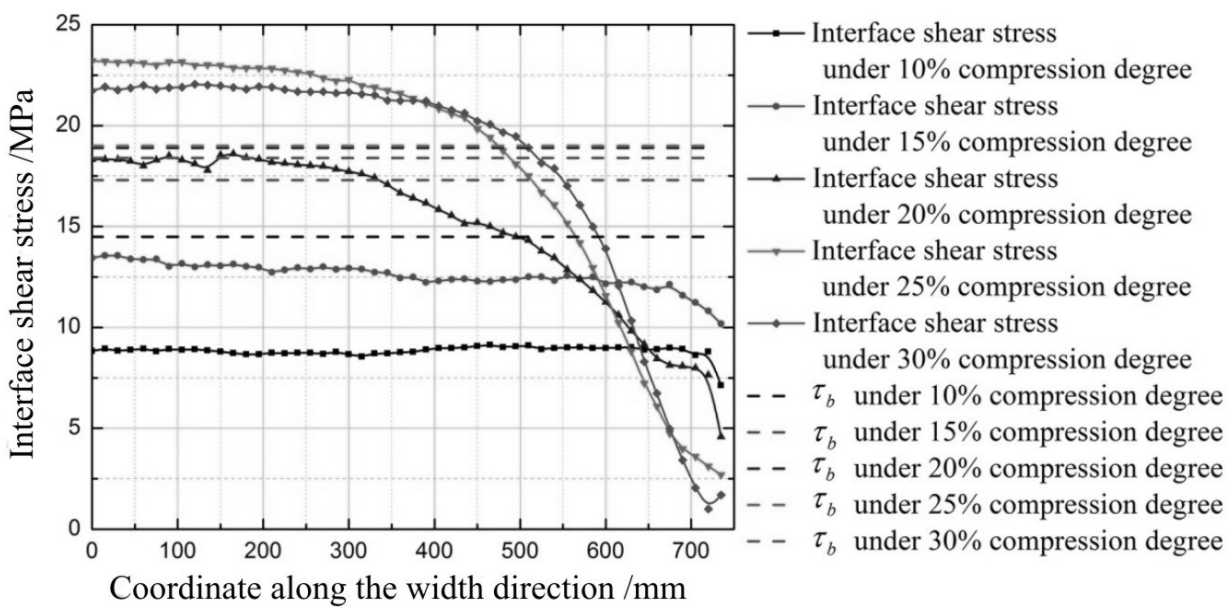

Fig. 9. Shear stress and of interface under different compression degrees.

interface area can be obtained. $K_{\text {damage }}$ can represent the size of the degree of damage associated with the bonding interface. The ratio $K_{S}=\tau_{\max } / \tau_{b}$ of the maximum shear stress $\tau_{\max }$ of the bonding interface during the rolling process to its tangential bond strength $\tau_{b}$ can be obtained, which can represent the risk factor of the damage occurring for the interface at a certain compression degree. The specific data are shown in Table 6.

It can be seen from Fig. 9 and Table 6 that when the compression degree of the first pass is more than $25 \%$, the bonding interface undergoes a wide range of damage, and the damage will occur at the position of the largest stress in the middle of the plate and the damage area will exceed $50 \%$, while no damage will occur for the interface when the compression degree is less than $20 \%$. However, when the compression degree reaches $20 \%$, the risk factor of the damage during the interfacial rolling process is close to 1 , which is easy to cause local damage due to the small fluctuation of the process parameters during the rolling process. On the one hand, taking into account the demand of the subsequent rolling pass on the interfacial bonding strength, we should try to improve the compression degree of the first pass to improve the interfacial bonding ratio $K_{B}$. On the other hand, too large shear stress should not be made during the first pass rolling process to cause the damage to the bonding interface; instead, it should be appropriate to reduce the compression degree of the first pass to ensure that there is a certain degree of security for the interface. Taking into account the above two factors, the compression degree of the first pass for this type of Hot rolled stainless steel clad plate should be set into the range of 15 to $20 \%$. In this process 
T a b 1 e 6

\section{Damage Coefficient $K_{\text {damage }}$ and Hazard Coefficient $K_{S}$ of Interface under Different Compression Degrees}

\begin{tabular}{|c|c|c|c|}
\hline $\begin{array}{c}\text { Compression } \\
\text { degree } \varepsilon, \%\end{array}$ & $K_{\text {damage }}$ & $\begin{array}{l}\tau_{\max }, \\
\mathrm{MPa}\end{array}$ & $K_{S}$ \\
\hline 10 & 0 & 9.0 & 0.62 \\
\hline 15 & 0 & 13.5 & 0.78 \\
\hline 20 & 0 & 18.4 & 0.98 \\
\hline 25 & 0.64 & 23.2 & 1.22 \\
\hline 30 & 0.70 & 21.9 & 1.19 \\
\hline
\end{tabular}

parameter range, it can not only guarantee a certain interfacial bonding binding rate of the first pass, but also ensure that the damage does not occur for the bonding interface during the rolling process.

\section{Conclusions}

1. The influence law of various factors on the bonding strength of the interface at high temperature during the rolling process of stainless steel clad plate was analyzed, and a phenomenological prediction model with high temperature bonding strength for the bonding interface was established.

2. The test experiment of the interfacial high temperature bonding strength was designed. The high temperature bonding strength of bonding interface for the stainless steel-carbon steel at 1200 and $1000^{\circ} \mathrm{C}$ was tested, and the interfacial bonding ratio was obtained. The experimental results show that there is a linearly increasing relationship between the interfacial bonding ratio and the compression degree.

3 . With the finite element numerical simulation and the bonding strength prediction model established in this paper, the interface bonding and damage state of the stainless steel clad plate of the typical specification were analyzed during the first pass rolling process. The results show that the interface would have a wide range of damage when the compression degree of the first pass is more than $20 \%$. The optimal compression degree range is 15 to $20 \%$.

Acknowledgments. The authors would like to acknowledge the financial support provided by the National High Technology Research and Development Program of China (No. 2013AA031302) and the Beijing Municipal Natural Science Foundation (No. 3154036) and the Fundamental Research Funds for the Central Universities of China (No. FRF-TP-16-010A3).

1. L. Li, K. Nagai, and F. X. Yin, "Progress in cold roll bonding of metals," Sci. Technol. Adv. Mat., 9, No. 2, 023001 (2008), DOI: 10.1088/1468-6996/9/2/023001.

2. M. Yang, X. Zuo, M. Zhao, and J. Wang, "Research progress of manufacturing technology for stainless steel clad plate hot," Work. Technol., 20, 027 (2012).

3. Y. H. Yang, G. Y. Lin, D. D. Chen, et al., "Fabrication of Al-Cu laminated composites by diffusion rolling procedure," Mater. Sci. Technol., 30, No. 8, 973-976 (2014).

4. R. Abedi and A. Akbarzadeh, "Bond strength and mechanical properties of threelayered st/az31/st composite fabricated by roll bonding," Mater. Design, 88, 880-888 (2015). 
5. H. Kim, G. T. Kang, and S. I. Hong, "Thermomechanical processing and roll bonding of tri-layered $\mathrm{Cu}-\mathrm{Ni}-\mathrm{Zn} / \mathrm{Cu}-\mathrm{Cr} / \mathrm{Cu}-\mathrm{Ni}-\mathrm{Zn}$ composite," Metall. Mater. Trans. A, 47, No. 5, 2267-2276 (2016).

6. M. M. Hoseini-Athar and B. Tolaminejad, "Interface morphology and mechanical properties of $\mathrm{Al}-\mathrm{Cu}-\mathrm{Al}$ laminated composites fabricated by explosive welding and subsequent rolling process," Met. Mater. Int., 22, No. 4, 670-680 (2016).

7. Z. J. Wang, L. Zhai, M. Ma, et al., "Microstructure, texture and mechanical properties of Al/Al laminated composites fabricated by hot-rolling," Mater. Sci. Eng. A, 644, 194-203 (2015).

8. M. Ma, P. Huo, W. C. Liu, et al., "Microstructure and mechanical properties of $\mathrm{Al} / \mathrm{Ti} / \mathrm{Al}$ laminated composites prepared by roll bonding," Mater. Sci. Eng. A, 636, 301-310 (2015)

9. W. N. Kim and I. H. Sun, "Interactive deformation and enhanced ductility of tri-layered Cu/Al/Cu clad composite," Mater. Sci. Eng. A, 651, 976-986 (2015).

10. J. S. Kim, J. Park, K. S. Lee, et al., "Correlation between bonding strength and mechanical properties in $\mathrm{Mg} / \mathrm{Al}$ two-ply clad sheet," Met. Mater. Int., 22, No. 5, 771-780 (2016).

11. X. B. Li, G. Y. Zu, and P. Wang, "High strain rate tensile performance and microstructural evolution of $\mathrm{Al} / \mathrm{Cu}$ laminated composite under dynamic loading," Mater. Sci. Eng. A, 612, No. 33, 89-95 (2014).

12. Y. Zhang, S. Ji, G. Scamans, and Z. Fan, "Interfacial characterisation of overcasting a cast Al-Si-Mg (A356) alloy on a wrought Al-Mg-Si (AA6060) alloy," J. Mater. Process. Technol., 243, 197-204 (2017).

13. H. Kim and I. H. Sun, "Deformation and FRACTURE OF DIFFUSION-BONDEd $\mathrm{Cu}-\mathrm{Ni}-\mathrm{Zn} / \mathrm{Cu}-\mathrm{Cr}$ layered composite," Mater. Design, 67, 42-49 (2015).

14. X. Li, G. Zu, and P. Wang, "Effect of Strain rate on tensile performance of $\mathrm{Al} / \mathrm{Cu} / \mathrm{Al}$ laminated composites produced by asymmetrical roll bonding," Mater. Sci. Eng. A, 575, No. 13, 61-64 (2013).

15. L. Meng, L. Zhang, Zhou, S. P. Yang, et al., "Effect of annealing temperature on separation strength of $\mathrm{Ag} / \mathrm{Cu}$ composite plate," Trans. Mater. Heat Treat., 23, No. 3, 31-34 (in Chinese) (2002).

16. S. P. Lu, H. M. Yang, J. S. Yu, et al., "Effect of composite rolling deformation on $\mathrm{Ag} / \mathrm{Cu}$ bondng interface," Rare Metals, 37, No. 2, 330-334 (in Chinese) (2013).

17. X. P., Zhang, T. H. Yang, S. Castagne, et al., "Proposal of bond criterion for hot roll bonding and its application," Mater. Design, 32, No. 4, 2239-2245 (2011).

18. Q. Qin, D. T. Zhang, Y. Zang, and B. Guan, "A simulation study on the multi-pass rolling bond of 316L/Q345R stainless clad plate," Adv. Mech. Eng., 7, No. 7, 1-13 (2015).

19. H. Wang, X. Cheng, $\mathrm{S}$. Fu, et al., "Influence of first reduction ratio on the interfacial bonding properties of composite plate of hot rolled stainless steel," Forg. Stamp. Technol., 41, No. 4, 19-24 (2016).

20. L. Li, X. J. Zhang, H. Y. Lin, and F. X. Yin, "Formation mechanism of oxide inclusion on the interface of hot-rolled stainless steel clad plates," J. Iron Steel Res., 25, No. 1, 43-47 (2013).

21. C. Y. Sun, L. Li, M. W. Fu, and Q. J. Zhou, "Element diffusion model of bimetallic hot deformation in metallurgical bonding process," Mater. Design, 94, 433-43 (2016). 
22. L. R. Vaidyanath., M. G. Nicholas., and D. R. Milner, "Pressure welding by rolling," Br. Weld. J., 6, 13-28 (1959).

23. H. A. Mohamed and J. Washburn, "Mechanism of solid state pressure welding," Weld. J., 54, 302-310 (1975).

24. X. P. Zhang, T. H. Yang, S. Castagne, and J. T. Wang, "Microstructure; bonding strength and thickness ratio of $\mathrm{Al} / \mathrm{Mg} / \mathrm{Al}$ alloy laminated composites prepared by hot-rolling," Mater. Sci. Eng. A, 528, Nos. 4-5, 1954-1960 (2011).

25. J. M. Parks, "Recrystallization in welding," Weld. J., 32, No. 5, 209s-221s (1953).

26. R. Jamaati and M. R. Toroghinejad, "Effect of friction, annealing conditions and hardness on the bond strength of Al/Al strips produced by cold roll bonding process," Mater. Design, 31, No. 9, 4508-4513 (2010).

27. P. He, J. C. Feng, and Y. Y. Qian, "A new model of interfacial physical contact in diffusion bonding," J. Mater. Sci. Technol., 20, No. 1, 109-112 (2004).

28. H. M. Ding, X. L. Fan, J. F. Wang, et al., "Interface characterization of hot-rolled stainless steel/carbon steel clad," Trans. Mater. Heat Treat., 32, No. 11, 18-22 (2011).

29. C. Hoppe, H. C. Schmidt, I. Hordych, et al., "Cold pressure welding by incremental rolling: deformation zone analysis," (2016), DOI: 10.1063/1.4963507.

30. J. Song, "Investigation into friction condition in finite element analysis of rolling problem," Lubr. Eng., 5, 17-21 (1992).

31. Q. J. Chen, Y. L. Kang, H. P. Hu, et al., "Simulation of rolling process for wide and thin plate of alloy steel by finite element method," J. Plast. Eng., 12, 163-167 (2005).

Received 15. 09. 2017 